\title{
PAPEL DE LA INTERCULTURALIDAD EN EL CONTROL DEL CÁNCER EN PUEBLOS INDÍGENAS
}

Lida Salazar', Mónica Benavides², Santiago Valencia ${ }^{3}$

Nada va bien en un sistema político en que las palabras contradicen a los hechos.

Napoleón Bonaparte

\section{Resumen}

Pese a su importancia, la población indígena se caracteriza por tener mayor pobreza, menor acceso a educación y a salud, lo que conlleva a reducir su expectativa de vida. Otros aspectos como el elevado nivel de desempleo, la precariedad de servicios sociales, la vulneración de Derechos Humanos y la degradación del ambiente, configuran un desfavorable escenario de discriminación, marginación y exclusión. Aunque es bien conocida la necesidad de ofrecer servicios de salud diferenciales, esto aún no logra materializarse, lo que sumado a la ausencia de información y estrategias apropiadas, lleva a la falta de goce efectivo de la población indígena de los servicios para el control del cáncer que son accesibles al resto de la población. Dado lo anterior, es indispensable la reflexión respecto al estado y papel de la interculturalidad en el control del cáncer. programas nacionales de salud, DeCS (BIREME).

\footnotetext{
${ }^{1}$ Bacterióloga. Magíster en Salud Pública. Grupo Área de Salud Pública. Instituto Nacional de Cancerología ESE. Isalazar@cancer.gov.co. 4320160 ext. 4406.

${ }^{2}$ Médica. Magíster en Salud Pública. Grupo de Prevención y Detección Temprana del Cáncer. Instituto Nacional de Cancerología- ESE. mrbenavides@cancer.gov.co 3 Psicólogo. Investigador Asociado independiente. santiagovalenciarico@gmail. com
} 


\title{
ROLE OF INTERCULTURALITY IN THE CONTROL OF CANCER IN TOWNS OF INDIGENOUS PEOPLE
}

Lida Salazar, Mónica Benavides, Santiago Valencia

\begin{abstract}
Despite its importance, the indigenous population is characterized by greater poverty, less access to education and health services, which leads to reduced life expectancy. Other facts such as the high rate of unemployment, the precarious social services, the violation of $\mathrm{Hu}-$ man Rights and the environment degradation, constitute an unfortunate scenario of discrimination, marginalization and exclusion. Although the need to offer differential health services is well known, this has not yet materialized, in addition, the lack of information and appropriate strategies, leads to indigenous population unable to enjoy effectively the cancer control services. which are accessible to the rest of the population. According to the above mentioned, it is essential to reflect on the condition and role of interculturality in cancer control.
\end{abstract}




\section{PAPEL DA INTERCULTURALIDADE NO CONTROLE DO CÂNCER EM POVOS INDÍGENAS.}

Lida Salazar, Mónica Benavides, Santiago Valencia

\section{Resumo}

Apesar de sua importância, a população indígena é caracterizada por maior pobreza, menor acesso à educação e à saúde, o que leva a uma redução da expectativa de vida. Outros aspectos, como o alto nível de desemprego, a precariedade dos serviços sociais, a violação dos direitos humanos e a degradação do meio ambiente, constituem um cenário desfavorável de discriminação, marginalização e exclusão. Embora a necessidade de oferecer serviços diferenciais de saúde seja bem conhecida, isso ainda não é uma realidade, o que, somado à falta de informações e estratégias adequadas, leva à falta de aproveitamento efetivo dos serviços de controle de câncer por parte da população indígena, embora tais serviços são acessíveis para o resto da população. Diante do exposto, é essencial refletir sobre o status e o papel da interculturalidade no controle do câncer. 


\section{Introducción}

Aunque la población indígena en Colombia no alcanza el 3\% de la población total, en general se ha caracterizado a nivel nacional e internacional por ser una de las comunidades más marginadas de la sociedad, con mayores niveles de pobreza, menor nivel de educación, menor expectativa de vida y tener las peores condiciones de salud. Otros factores que empeoran su situación son tasas de desempleo significativamente más elevadas, servicios sociales no disponibles o de difícil acceso, violación de derechos humanos, desplazamientos, debido a conflictos armados, y degradación del ambiente.

Sumado a este panorama, la falta de registro de los niveles de incidencia, prevalencia y mortalidad de las poblaciones indígenas con cáncer, limitan el desarrollo de estrategias que impacten favorablemente el control de esta patología en dichas poblaciones. Adicionalmente, aunque se ha observado la necesidad de ofrecer servicios de salud con un enfoque diferencial a las comunidades indígenas, este hecho todavía no ha logrado materializarse. Situaciones que conllevan a su pobre participación y falta de empoderamiento en el desarrollo de acciones frente al cáncer, a la perpetuación de la marginalidad, la discriminación y la falta de goce efectivo de la población indígena de los servicios para el control del cáncer que son accesibles al resto de la población. Atendiendo a estas limitaciones del contexto, se hace necesario, por una parte, reflexionar acerca de la suficiencia, oportunidad, pertinencia y eficiencia de las acciones de la salud pública direccionadas a mitigar las necesidades de la población indígena colombiana; y en segundo lugar sugerir los aportes de la intercultural en la formulación de una estrategia para al control del cáncer en dicha población.

\section{Pueblos indígenas}

Las poblaciones indígenas denominadas también primeros pueblos, pueblos tribales, aborígenes y autóctonos, no disponen hasta la actualidad de una definición especial aceptada globalmente; sin embargo se ha considerado hacer uso del concepto "pueblos indígenas" adoptado por el sistema de Naciones Unidas (12) y que abarca a los pueblos que:

- Se identifican a sí mismos y son reconocidos y aceptados por su comunidad como indígenas.

- Tienen continuidad histórica demostrada con sociedades pre-coloniales $o$ anteriores al asentamiento de otros pueblos.

- Tienen fuertes lazos con sus territorios y sus recursos naturales.

- Poseen sistemas sociales, económicos o políticos distintos.

- Conservan lenguas, culturas y creencias distintas.

- Forman grupos no dominantes de la sociedad.

- Están dispuestos a mantener y reproducir sus entornos y sistemas ancestrales como pueblos y comunidades diferenciados.

Se considera que hay por lo menos 5000 grupos indígenas compuestos de unos 370 millones de personas que viven en más de 70 países de los cinco continentes (3). En las Américas habitan de 45 a 
50 millones de indígenas pertenecientes a más de 600 pueblos, representando casi $10 \%$ de la población total y $40 \%$ de la población rural de América Latina y el Caribe (1). Aunque estos pueblos representan una importante pluralidad de culturas, religiones, tradiciones, lenguas e historias, se encuentran entre los grupos de población más marginados y su estado de salud es notoriamente diferente del de las poblaciones no indígenas (4). Este patrón se observa tanto en países desarrollados y no desarrollados -aun cuando los pueblos indígenas son la mayoría de población nacional- $\mathrm{y}$ ha sido históricamente construido por factores políticos, económicos, sociales, militares y ambientales, que originan privación material, jurídica y simbólica, y de reproducción de relaciones de desventaja (5).

Los indicadores de salud, que van desde la mortalidad materna hasta los partos hospitalarios y cobertura de vacunas son peores entre los pueblos indígenas $y$ sus niveles de ingreso, al igual que sus indicadores de desarrollo humano como educación y salud, han quedado rezagados en comparación con los del resto de la población $(6,7)$. Se cuentan entre sus causas de muerte más comunes malaria, oncocercosis, infecciones respiratorias agudas, tuberculosis, enfermedades diarreicas, desnutrición, alcoholismo, drogadicción, enfermedades crónicas y degenerativas, VIH / sida y suicidio; y aunque los saberes, las prácticas, los terapeutas de la medicina indígena $y$ los recursos comunitarios son de gran cuantía para el mantenimiento general de la vida, ante un perfil epidemiológico tan complejo cubren parcialmente sus necesidades de salud (1).

\section{Pueblos indígenas y legislación}

Colombia tiene una profusa normatividad que reconoce a los pueblos indígenas. El más relevante es el Convenio 169 de 1989 de la Organización Internacional del Trabajo (OIT) sobre los derechos de los Pueblos Indígenas y Tribales en Países Independientes ratificado mediante la Ley 21 de 1991; dicho Convenio tiene dos postulados básicos: el derecho de los pueblos indígenas a mantener y fortalecer sus culturas, formas de vida e instituciones propias, y su derecho a participar de manera efectiva en las decisiones que les afectan (8). Este Convenio tiene sus referentes en diferentes convenciones de las Naciones Unidas y obedece a la paulatina consideración de situaciones específicas de grupos humanos que a pesar de la Declaración Universal de los Derechos Humanos no gozan de los derechos y libertades fundamentales de la misma manera que otros (9). Así mismo, se ha propiciado un proceso de reafirmación cultural y conciencia de la identidad de los pueblos indígenas, mediante el reconocimiento del país como pluriétnico y multilingüe en la Constitución Política de 1991, con cerca de 30 artículos referidos a los grupos étnicos $\mathrm{y}$ a sus diversas y particulares culturas.

\section{Pueblos indígenas de Colombia}

Para 2005, según el Departamento Administrativo Nacional de Estadística (DANE), en Colombia, la mayoría de la población indígena se ubica en el área rural en regiones como la selva, las sabanas de la Orinoquia, los Andes colombianos, los valles interandinos y la planicie del Caribe; siendo los departamentos con mayor porcentaje de indígenas Guainía, 
Vaupés, Amazonas, Vichada, La Guajira, Cauca y Nariño -estos tres últimos concentran aproximadamente la mitad de los indígenas del país (10). Sin embargo, en las cabeceras municipales y en las grandes ciudades del país reside una minoría de indígenas, resultado del agotamiento de las tierras en los resguardos y desplazamiento forzado (11).

Según el censo oficial del país, en el año 2005 residían 1.392.623 indígenas, que representaban el 3,43\% del total de la población nacional (15) organizados en 87 pueblos y 700 resguardos indígenas, aunque la Organización Nacional Indígena de Colombia (ONIC) ha manifestado en diferentes oportunidades que esta cifra asciende hasta los 102 (12), entre los cuales no se han identificado los niveles de incidencia, prevalencia y mortalidad en cáncer en general o dicha información no se encuentra disponible más allá de cifras aisladas sobre tipologías, pueblos y regiones particulares (13).

No obstante, se ha podido establecer que el cáncer es la segunda causa de mortalidad en personas indígenas entre 45 y 59 años de edad y en los niños es una causa importante de muerte, especialmente la leucemia (14); datos que no son comparables con los de la población infantil en general, obedeciendo a factores tales como: el subregistro de información, la forma de comprender el cáncer y las barreras existentes para la prestación del servicio de salud, desde el diagnóstico hasta el tratamiento integral de manera oportuna. También se ha encontrado que las condiciones socioeconómicas influyen en la supervivencia al cáncer (15), hecho que afecta frontalmente a las comunidades indígenas.

\section{Concepto indígena de salud}

Cada pueblo indígena tiene su forma particular de explicar el mundo que lo rodea y formas propias de representar y entender los procesos salud-enfermedad. El concepto de salud, en estos pueblos es verdaderamente integral $y$ se relaciona con el manejo del mundo y del medio ambiente (16). En la cosmovisión de los pueblos indígenas, la salud ha sido definida como: "el estado de equilibrio armónico de las relaciones de la persona consigo misma, con su familia, la comunidad, el territorio y se expresa en la relación con el territorio y su cuidado, con el ambiente y las relaciones sociales, la autoridad, el respeto, la colectividad, la producción y alimentación, las relaciones con otros pueblos, culturas y con el estado". Por otra parte, las enfermedades son consecuencia del desequilibrio en la naturaleza o en el comportamiento personal generado por un mal relacionamiento con el entorno que lo lleva a la desarmonía (17). Las comunidades también otorgan un significado especial a los usos que hacen de su territorio o las prácticas sociales relacionadas con la salud individual y la de la comunidad. Por lo tanto, el bienestar de un individuo está unido al de la comunidad y el entorno, a través de prácticas que buscan un equilibrio espiritual entre individuos, comunidades y su entorno (18).

\section{Población indígena y salud}

En términos de salud debe señalarse que para el país solo hasta la Ley 691 de 2001 hubo claridad acerca de la vinculación de los pueblos indígenas al Sistema General de Seguridad Social en Salud. En 
esta se incorpora la población al régimen subsidiado, y se otorga la posibilidad de que las organizaciones indígenas debidamente soportadas conformen entidades promotoras de salud e instituciones prestadoras de servicios de salud propias (19). Distintos instrumentos (Tabla 1) posteriores destacan la necesidad de introducir la interculturalidad como un eje central de las acciones de salud tanto del Plan Obligatorio de Salud como del Programa de Intervenciones Colectivas, siendo materializado en el Plan Decenal de Salud Pública, en los componentes especiales de aceptabilidad e interculturalidad de la Ley Estatutaria en Salud, y más recientemente, con la reglamentación del Sistema Indígena de Salud Propia e Intercultural - SISPI - en el Decreto 1953 de 2014.

Tabla 1. Legislación en salud población indígena en Colombia.

\section{Normas}

Ley 89 del 25 de noviembre de 1890

Resolución 10013 de 1981

Resolución 5078 de 1982

Decreto 1811 de 1990

Política de 1991 y Ley 21 de 1991

Decreto 1088 de 1993

\section{Referentes}

Determina la manera cómo deben ser gobernados los salvajes que vayan reduciéndose a la vida civilizada. Organización de cabildos y resguardos indígenas.

Que los programas de prestación de servicios de salud (AP) que se desarrollan en las comunidades indígenas, deben adaptarse a la estructura organizacional, política, administrativa y socioeconómica, con el fin de respetar sus valores tradicionales, creencias, actitudes y acervo cultural.

Establece la promoción y el intercambio de conocimientos entre los médicos tradicionales indígenas y el personal de la salud de la sociedad hegemónica.

Reglamenta parcialmente la Ley 10 de 1990. Estableció el derecho a las comunidades de los grupos y pueblos étnicos indígenas a participar en los procesos de diagnóstico, formulación y elaboración de planes, programas y proyectos, toma de decisiones, administración y gestión relacionada con los servicios de salud, así como la necesidad que estos procesos fueran previamente acordados con ellos.

Ratifica el Convenio 169 de 1989 de la Organización Internacional del Trabajo. Establece que los gobiernos deberán velar por la existencia de servicios de salud adecuados a dicha población o proporcionar los medios para que dichos grupos y pueblos puedan organizar y prestar tales servicios bajo su responsabilidad y control, así como la necesidad de su planeación y administración en cooperación conjunta entre el Estado y las organizaciones. La ley establece el derecho a la consulta previa por parte de los grupos y pueblos étnicos indígenas.

Regula la creación de las asociaciones de Cabildos y/o autoridades tradicionales indígenas. 
Decreto 330 de 2001

Ley 691 de 2001

Acuerdo 326 de 2005

Decreto 4972 de 2007
Construcción y funcionamiento de Entidades Promotoras de Salud, conformadas por cabildos y/o autoridades tradicionales indígenas.

Reglamenta la participación de los Grupos Étnicos en el Sistema General de Seguridad Social en Salud (SGSSS)

Adoptan algunos lineamientos para la organización y funcionamiento del régimen Subsidiado de los pueblos indígenas. Afiliaciones colectivas, traslados colectivos, adecuaciones del Plan Obligatorio (M.T, subsidio alimentario, casas de paso y guías bilingües).

Reglamentan las instituciones prestadoras de servicios de salud indígenas.

Fuente: Autoridades Tradicionales Indígenas de Colombia, 2016.

\section{Sistema Indígena de Salud Propia e In- tercultural (SISPI)}

De acuerdo con Sandra Land de la Organización Mundial de la Salud (OMS) "cada pueblo indígena tiene sus creencias y prácticas únicas en lo referente a la salud, así como sus propios recursos comunitarios para la promoción de la salud, la prevención de enfermedades o la cura de los males comunes" (20). Esto ha hecho que la medicina tradicional mantenga su vigencia en los pueblos indígenas; recurriendo al empleo de plantas medicinales y otros recursos terapéuticos como los rituales, los cantos, las ceremonias y a la práctica de los proveedores tradicionales de salud como parteros o sobanderos. Esta forma de lograr la salud exige de una política centrada en la comunicación, un estilo de relación que valoriza el saber local, en el que individuos como el jefe de la comunidad, médicos tradicionales, chamanes, curanderos, parteras, hueseros, consejo de ancianos, líderes y promotores, todos con sus saberes y experiencias, constitu- yen una red de autoridades legitimadas por su comunidad para el cuidado de sus destinos, incluyendo el cuidado de la vida, salud y el desarrollo de los individuos y sus entornos (6).

Desde este contexto, en Colombia muchas de las actuales organizaciones indígenas se encuentran en el proceso de construir su SISPI, el cual incluye la materialización de la atención intercultural en salud, la incorporación del concepto salud-enfermedad como una construcción de cada sistema cultural y la comprensión de la existencia de enfermedades que son propias de las culturas indígenas y que requieren un abordaje diagnóstico y terapéutico desde su lógica propia para lograr restablecer la salud. En resumen, el SISPI, es el conjunto de políticas, normas, principios, recursos, instituciones y procedimientos, que tiene como base la sabiduría ancestral, la madre tierra y la cosmovisión de cada pueblo, en complementariedad del SGSSS y que se crea haciendo un énfasis especial en la diferenciación de la salud 
en las personas de la población civil en general y la salud de los pueblos indígenas, como resultado de las disímiles características étnicas, culturales, cognoscitivas, que hacen indispensable que se garantice su medicina propia (21).

Por otra parte, el carácter de 'propio' en el SISPI, está dado por la consulta previa, herramienta fundamental del derecho de los pueblos indígenas a la autodeterminación, que tiene por objeto garantizar a estos grupos la coparticipación en las decisiones administrativas y legislativas que los afecten como: la elaboración e implementación de leyes y normas, programas y proyectos de desarrollo, proyectos de explotación de recursos y de infraestructura en sus territorios (22). El vínculo de la consulta previa consiste en un diálogo de buena fe, libre de presiones o manipulaciones, en el que las partes deben cooperar para intentar llegar a decisiones consensuadas e informadas, buscando amparar los derechos indígenas de propiedad sobre sus tierras, recursos y territorios, además de otros derechos y a establecer sus propias prioridades para el desarrollo, la salud y bienestar físico (24). La base jurídica del derecho a la consulta es el Convenio 169 sobre Pueblos Indígenas y Tribales en Países Independientes, de la OIT de 1989 que a finales de 2012, fue ratificado por 15 Estados de América Latina; en el país fue incorporado en la legislación nacional por la Ley 21 de 1991 (24).

Aunque se considera que Colombia es el país con más larga y amplia pericia en dicho tema, pues ya desde 1994, el Estado ejecutaba consultas previas y solo en el primer cuatrimestre del año 2012, adelantó 470 protocolizaciones con grupos étnicos en 65 procesos de consulta previa, en su mayoría en los sectores mi- nero, energético y ambiental (25) y en el ámbito de la salud, existen sentencias de la Corte Constitucional (4) que en concordancia con el Convenio 169, confirman que la intervención a los pueblos indígenas se debe hacer cumpliendo siempre con el requisito de la consulta previa $(26,27)$, casos documentados de consultas previas en temas de salud no fueron encontrados.

\section{Cáncer y población indígena}

La Agencia Internacional para la Investigación en Cáncer (IARC) sostiene que las poblaciones indígenas tienen mayores tasas de incidencia de tumores que se consideran prevenibles, como los asociados al tabaco, como el de pulmón o los de cabeza y cuello, o a la presencia de infecciones, como el de estómago, hígado o cáncer de cérvix; esto basado en una comparación realizada con las tasas de incidencia de cáncer obtenidas de los registros poblacionales, entre el 2002 y 2006, de tres estados de Australia (Queensland, Australia Occidental y el Territorio del Norte), Nueva Zelanda y las áreas de prestación de servicios de salud contratados en Estados Unidos. En ambas poblaciones, tanto indígenas como no indígenas, los tumores más comunes entre los hombres eran los de pulmón, próstata y colorrectal, y entre las mujeres los de mama, pulmón y colorrectal (28).

En los Estados Unidos, los indoamericanos y los nativos de Alaska (I/NA) tienen patrones de cáncer únicos debido a su historia y cultura, el lugar donde viven y la forma en que obtienen atención médica. En los últimos 20 años, las tasas de mortalidad por cáncer se redujeron 
más rápidamente entre las personas de raza blanca, quienes además vivían más tras el diagnóstico de casi todos los tipos de cáncer con respecto a los I/NA. Estas disparidades, probablemente se relacionen con los factores sociales, conductuales y ambientales que ponen a las personas en mayor riesgo de contraer cáncer, y a la falta de acceso de aquellas personas con cáncer a servicios médicos de calidad para las pruebas de detección, el diagnóstico y el tratamiento. Las cinco principales causas de muerte por cáncer entre los hombres I/NA fueron cáncer de pulmón, colon y recto, próstata, hígado y riñón. Las cinco causas principales de muerte por cáncer entre las mujeres de estas poblaciones fueron cáncer de pulmón, mama, colon y recto, páncreas y ovario (29).

Para el caso de Colombia no hay registros disponibles, pero se estima que en La Guajira, donde el 45 \% de la población es indígena, se encuentran menores tasas de cáncer que para el resto del país (62 / 100.000 frente a $167 / 100.000$, respectivamente). Así mismo, el riesgo de morir de cáncer de cuello uterino es más alto en la región amazónica, y las tasas de mortalidad de cáncer de estómago fueron más altas en el Cauca, ambas regiones con un nivel muy alto de población indígena (20). De acuerdo con el Ministerio de Salud y Protección Social a partir de los datos del Sistema de Vigilancia de Salu Pública (SIVIGILA), entre los años 2009 y 2014 se presentaron solo 71 casos de cáncer en población indígena, de estos, 53 correspondieron a leucemia aguda y los restantes a cáncer infantil (30).

Aunque sabe que la carga global del cáncer tiende a ser menor en las personas indígenas que en el resto de la población, se prevé un aumento global de la incidencia de esta patología en las próximas décadas y aunque los programas de lucha contra el cáncer cuenten ya con numerosos elementos, es necesario conocer mejor la magnitud y el perfil del cáncer entre la población indígena para poner en marcha políticas de control dirigidas a reducir la carga de esta enfermedad a nivel mundial. Además, es posible que la cobertura de la población sea desigual y que determinados grupos, como los habitantes de zonas rurales, la población indígena y los emigrantes recién llegados, encuentren dificultades de acceso a los servicios (31).

\section{Interculturalidad en salud}

La Organización Panamericana de la Salud (OPS) sostiene que la interculturalidad "puede ser entendida como la habilidad para reconocer, armonizar y negociar las innumerables diferencias que existen al interior de cada sociedad, además de la forma de interacción comunicativa que se produce entre los dos grupos humanos de diferentes culturas" (32). Por otra parte, la Organización Mundial de la Salud aprobó en 1993 la Iniciativa de Salud de los Pueblos Indígenas, en la que los Estados Miembros (5) se comprometieron a trabajar con la población indígena, por el objetivo de mejorar su salud y bienestar, reconociendo el valor del patrimonio cultural, su saber ancestral y la necesidad de conservarlos (32).

En el territorio nacional coexisten múltiples grupos y pueblos étnicos, lo cual hace del país un territorio multicultural 
caracterizados por distintos grupos étnicos, esto reconocido por la Constitución Política de Colombia de 1991 en la que se "reconoce y protege la diversidad étnica y cultural de la nación colombiana" (32). Ahora bien, el encontrarse frente a la coexistencia de diversas creencias, conocimientos, percepciones y prácticas, que son validadas socialmente por quienes las sustentan, permite la oportunidad de generar una dinámica entre las culturas con interacciones de reciprocidad, voluntad y horizontalidad en espacios de encuentro para negociar y otros para mantener las particularidades, enmarcado este en respeto a las diferencias, con el propósito de mejorar la salud de ambas partes (32).

De igual manera esa construcción de con-ciudadanía de respeto y de estima (33) fortalece los procesos de co-creación que van abriendo y afianzando relaciones de confianza, reconocimiento mutuo, comunicación efectiva, dialogo, debate, aprendizaje e intercambio de visiones particulares de entender el mundo y el actuar frente a él (34), lo que permite que la construcción de acciones en pro del mejoramiento de las calidades de salud de una comunidad sean basadas en la comprensión del contexto de la misma, siendo así más fácil que sean reconocidas, legitimadas y acopladas en sus dinámicas y seguramente alcanzarán un mejor impacto en términos de lo esperado.

\section{Estrategia intercultural para el control del cáncer en pueblos indígenas co- lombianos}

Prevenir y curar enfermedades crónicas, como el cáncer, representa un gran para el campo médico, dada su inmersión en el modelo biomédico que minimiza o margina la importancia del contexto social y cultural (el medio, la sociedad y la cultura del enfermo y de la enfermedad) en la génesis de la enfermedad, que recurre a estrategias ideológicas como la 'medicalización' de aspectos de la vida cotidiana -embarazo, nacimiento, crianza, sexualidad, vejez o muerte - y que ha aspirado alcanzar la máxima eficiencia diagnóstica, pronostica y terapéutica, usando una comprensión de la salud reducida y parcial, con la cual el uso de la tecnología y la sobreexposición farmacológica se asocia a buenos efectos terapéuticos; independientemente del resultado y de los éxitos obtenidos y demostrados sobre el paciente (35).

$\mathrm{Y}$ aunque, existen esfuerzos desde las ciencias básicas y se han realizado grandes avances en campos como el epidemiológico, los aspectos sociales y culturales asociados al cáncer no han sido lo suficientemente estudiados y son presentados como elementos de segundo orden, al momento de construir estrategias de salud pública para su control. Es por esto que la inclusión de la interculturalidad en el proceso de atención se convierte en un fenómeno que trasciende lo exclusivamente étnico, pues implica valorar la diversidad biológica, cultural y social del ser humano como un factor importante en todo proceso de salud y enfermedad (36).

El proceso de formular una estrategia intercultural para el control del cáncer en pueblos indígenas colombianos requiere transformaciones estructurales de largo plazo y responsabilidades que 
salud, pues son las estructuras del Estado en conjunto con las sociedades indígenas, quienes deben establecer las bases para un proyecto multicultural de sociedad, no desde el escritorio sino de los propios territorios. Sin embargo, en la literatura se han esbozado estrategias en salud de forma general que pueden contribuir a este proceso. Se ha descrito la necesidad de desarrollar acciones en tres niveles (37):

1. Acciones de comunicación intercultural, consideradas como factores que disminuyen las barreras sociales y culturales entre equipos de salud y usuarios. El mejorar los procesos de comunicación y diálogo, promueve una mayor comprensión de las expectativas de ambos actores y a la vez mejora el nivel de satisfacción de los usuarios con el sistema de salud biomédico. Es reconocido que aunque el lenguaje puede ser una gran barrera en la comunicación en sus aspectos gramaticales, simbólicos y semánticos, como tal no constituye un obstáculo infranqueable en la relación médico-paciente, si los profesionales están abiertos al diálogo y conocen en parte la cultura médica de sus pacientes. Esta acción se puede lograr a través de un proceso compuesto de cuatro elementos: "escuchar, explicar, reconocer y recomendar"(38). En este sentido, la comunicación educativa como una estrategia que contribuye a reducir la incidencia y la mortalidad por cáncer, así como a mejorar la calidad de vida de los pacientes y que se ha definido como un proceso que promueve el diálogo entre las personas que les permita ganar capacidades para la toma de decisiones autónomas, respecto a la salud, el cuerpo y la vida, y construir, participativamente, condiciones que posibiliten el ejercicio de sus derechos (39), puede también contribuir.

2. Acciones de validación cultural entre pacientes y profesionales. La validación cultural consiste en aceptar la legitimidad del modelo de salud $y$ enfermedad del paciente, considerando el contexto cultural en que este modelo emerge. La validación cultural no significa que el profesional comparta el mundo simbólico del paciente, sino que comprenda, respete e incluso integre algunos elementos culturales que considere relevantes para el proceso de recuperación del enfermo.

3. Acciones de negociación cultural ocurre cuando las creencias culturales y las expectativas de los pacientes no se contraponen a las del profesional de salud, interfiriendo en la confianza y adherencia al proceso terapéutico, y se logra una complementariedad entre los dos sistemas médicos: el occidental y el tradicional indígena, de tal manera que se respetan los conocimientos del otro y se generan espacios de confianza.

Dentro de este marco de acciones, la figura de facilitador intercultural toma fuerza, al convertirse en el vínculo entre el equipo de salud y las comunidades indígenas, a la vez que pertenece a las comunidades y es avalada por estas mismas. Lo que implica finalmente potenciar lo común entre el sistema médico occidental y el sistema médico indígena, respetando y manteniendo la diversidad 
(40). También, la formación de recurso humano en salud con orientación intercultural supone mejoras en las competencias técnicas y humanas, incidiendo en el respeto, el trato de los usuarios, el reconocimiento de las tradiciones culturales, para hacer frente a la exclusión en la atención y en búsqueda de la equidad en salud. Aunado a esto, la reafirmación de la importancia de la tradición indígena sobre plantas medicinales y tratamientos tradicionales exige su inclusión en la formación académica de los trabajadores de la salud y en las políticas de atención sanitaria intercultural, pues su abordaje dentro del espacio médico-académico posibilita la convergencia de ambos sistemas de conocimiento para el beneficio de la población indígena.

Por otra parte, dado que se requiere lograr una interculturalidad que vaya más allá de la reproducción del modelo asistencial moderno de salud en la población indígena, y que más bien sea la puesta en marcha de un paradigma autónomo de rescate y empoderamiento de la medicina tradicional y los saberes ancestrales en salud; que no lleve a la destrucción de quien es culturalmente distinto o a su disminución y asimilación, como sucede en las sociedades neocoloniales o a la simple tolerancia sin un verdadera inclusión, es necesario hacer claridad sobre los enfoques el paradigma intercultural en la atención de la salud (41):

1. Enfoque paternalista o integracionista: Se deriva de las políticas de integración, asimilación o indigenista. Aunque reconoce la diversidad, plantea medidas verticales de arriba hacia abajo y desde afuera, por lo que dentro de este enfoque el personal de salud es el experto y las comunidades solamente reciben conocimientos.

2. Enfoque culturalista: Reconocimiento del pluralismo cultural que afirma el derecho a la diferencia cultural y al valor intrínseco de cada cultura desde la visión que ella tiene de sí misma; otorga mayor grado de importancia a la lengua, los aspectos didácticos y lingüísticos. La forma de participación que promueve se reduce a consultas sin previa información y con base en metodologías diseñadas por autores externos. No interesan los procesos pedagógicos, ni la cosmovisión de los pueblos y comunidades involucradas en la organización de programas y proyectos de salud.

3. Enfoque de convivencia: Se parte del hecho de que es imprescindible el conocimiento del otro para que exista la convivencia. Se plantea para la coexistencia armónica entre los distintos grupos étnicos y se parte de que es importante la difusión del conocimiento adecuado a cerca de las culturas originarias para valorarlas y conocerlas. Con este enfoque se corre el riesgo de promover solamente la integración a partir del conocimiento mutuo sin avanzar hacia una relación de poder más equitativa.

4. Enfoque autonómico o empoderamiento: Parte del ejercicio de los derechos de los pueblos y comunidades hacia una relación más equitativa de poder. En este enfoque, la salud se deriva de los derechos sustantivos de los pueblos y comunidades, res- 
petando sus representaciones sociales. Promueve la organización de los servicios de salud que surjan de las comunidades; afianza la identidad colectiva y participación en las decisiones desde la organización hasta la implementación, evaluación y seguimiento de los servicios de salud. $\mathrm{Su}$ práctica permite convertir a los sujetos sociales en actores sociales, por lo que busca su inclusión y visibilización.

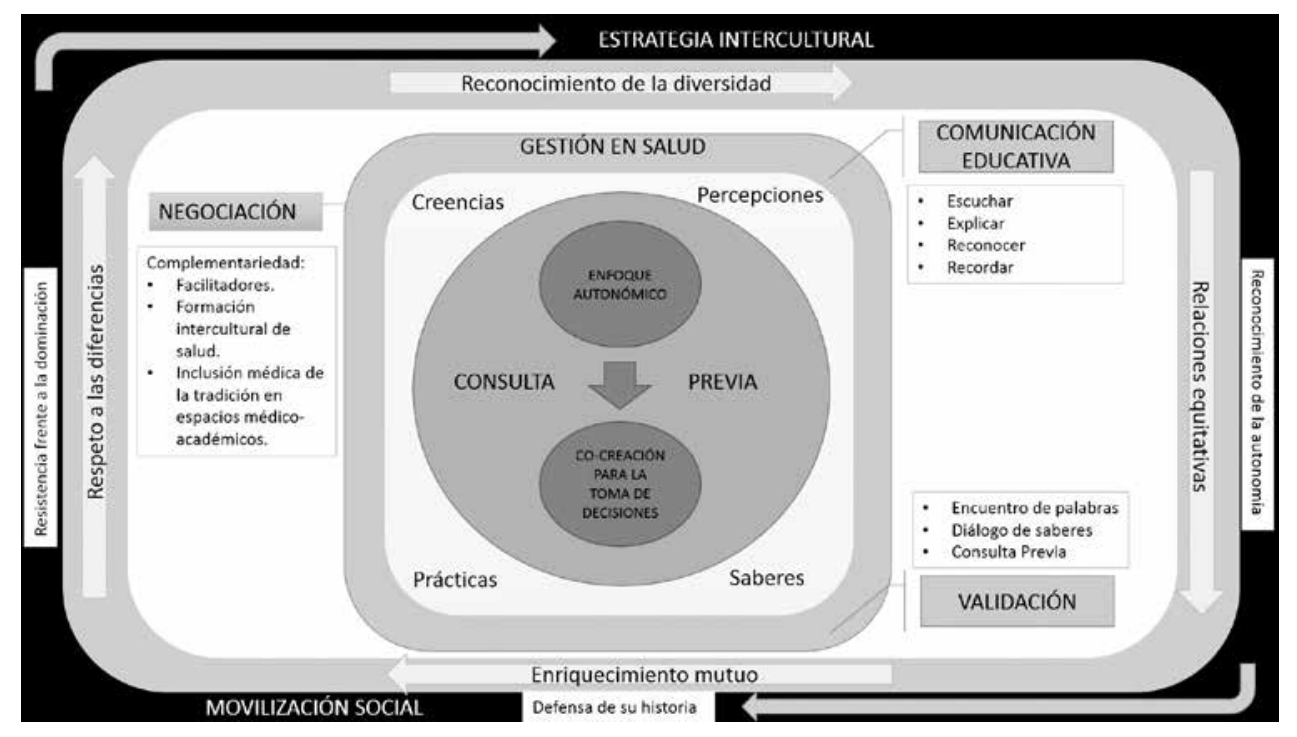

Figura 1. Estrategia de la participación intercultural en salud para el control del cáncer. Fuente: Equipo investigador.

Es de esperar que sea este último enfoque, la clave para el reconocimiento de espacios de comunicación, validación y negociación desde relaciones simétricas de poder que obliguen a cada sujeto a adoptar una postura de crítica y autocrítica para pensar, elegir, decidir y actuar. Del mismo modo, el enfoque autonómico es la mejor herramienta para conducir a las comunidades culturalmente diversas hacia la gestión de la salud desde su punto de vista, es decir, desde sus creencias, conocimientos, percepciones, saberes, prácticas y en diálogo perma- nente con los actores sociales y culturales que son distintos de su realidad a nivel político, social y cultural.

A diferencia de la población general, la población indígena se ha diferenciado a lo largo de la historia, por hacer uso frecuente de la movilización social como herramienta para el reconocimiento de su autonomía, sus autoridades y formas propias de organización, de resistencia frente a la dominación y el despojo de sus tierras, en defensa de su historia, cultura y tradición y de recuperación e impulso 
de la medicina indígena y la exigencia de programas de salud acordes con las características sociales y culturales de las comunidades (42).La movilización social es una de las manifestaciones más significativas en el proceso de incentivar a la sociedad a participar activamente en la solución de las problemáticas que la afectan; actúa dentro del contexto de la participación y brinda a los ciudadanos la posibilidad de tener intervención directa en los asuntos de interés general. Así mismo, permite la transformación de los ámbitos económico, social, político y cultural, y refleja las contradicciones y síntesis de los acontecimientos de una sociedad. Por lo que dentro de una estrategia para el control del cáncer en esta población, se debe continuar haciendo uso de este mecanismo de una forma transversal.

\section{Discusión de resultados}

Aunque los indígenas colombianos han tejido relaciones con sectores sociales e instituciones del Estado, desde hace años en un intento por encontrar puntos comunes sobre los cuales construir una serie de acciones que les permitan hacer frente a sus múltiples problemáticas de salud, y en general han trabajado en comisiones con autoridades regionales en temas de salud, educación o seguridad alimentaria; esto no ha sido suficiente para el desarrollo de acciones específicas frente al cáncer, patología considerada problema de salud pública, desde herramientas y estrategias que permitan el control del riesgo, la participación en el diagnóstico y la detección temprana, la aceptación y adherencia al tratamien- to y el cuidado paliativo, basadas en el empoderamiento comunitario con enfoque intercultural, que valore su lenguaje, modelo de organización social y comunitario, percepciones de la vida y sus propias respuestas al problema.

Atendiendo a estas limitaciones del contexto, se pone de manifiesto la necesidad de propuestas para la formulación de lineamientos o metodologías para el control del cáncer en poblaciones indígenas colombianas mediante la construcción participativa de una estrategia que no solo tenga en cuenta sino que además valore y capitalice los saberes, prácticas, creencias y costumbres locales; partiendo de un accionar inicial con cada una de las especificidades de los 90 pueblos indígenas del territorio nacional, desde su cosmovisión y forma de comprender la salud-enfermedad con una metodología netamente cualitativa y participativa que resalte el valor de la oralidad como elemento central de la comunicación de los pueblos indígenas y herramienta de resistencia en la preservación de su cultura y saberes ancestrales; de tal manera que lo explorado allí muestre elementos que permitan orientar $y$ facilitar la construcción de acciones en salud que sean reconocidas, legitimadas y acopladas por las comunidades a fin de alcanzar los objetivos propuestos.

Si bien el Estado a través de diferentes planes ha observado la necesidad de ofrecer servicios en salud con un enfoque diferencial a las comunidades indígenas, este hecho todavía no ha logrado materializarse, más aun, la referida ausencia de cifras e información en materia de salud es solo una pequeña muestra, más allá de esto, la presta- 
ción del servicio no es pertinente, pues "a pesar de su presencia histórica y de sus invaluables contribuciones, los pueblos indígenas son sumamente vulnerables en los países donde habitan y sus derechos humanos, al igual que sus derechos a la igualdad social, política y económica, están comprometidos o bien han sido negados. Como resultado, existen profundas inequidades en sus condiciones de vida, estado de salud y cobertura de servicios de salud" (43).

Por otra parte, las aproximaciones institucionales a las comunidades indígenas se han caracterizado por ser pobre, reconociendo su cosmogonía y su débil participación en el desarrollo de acciones frente a enfermedades como el cáncer, por lo que a la fecha, estas comunidades aún no gozan en su totalidad de un sistema de salud apropiado para sus creencias, usos y costumbres. Aunque se debe resaltar que se han hecho esfuerzos por entenderlos e incorporarlos en el abordaje de algunos problemas de salud, prueba de esto es la experiencia en Colombia de la Estrategia DOTS/ TAS para el manejo de la tuberculosis en pueblos indígenas, ubicados en los departamentos del Amazonas, Cesar, Nariño, Guainía, Guaviare, la Guajira y Vichada (47); reconociéndolos como socios trascendentales, aún existen falencias en el diálogo intercultural y en el reconocimiento y legitimación de sus estructuras sociales y organizacionales, dejando entrever que sin su participación en los procesos de intervención, es imposible alcanzar resultados óptimos en el mejoramiento de salud y de calidad de vida (48).
Esta paradoja de una racionalidad occidental en el desarrollo de políticas de salud para pueblos indígenas resulta ser causa y efecto del etnocentrismo en las estrategias de movilización social, que indefectiblemente conlleva a la perpetuación de la marginalidad, la discriminación y la falta de goce efectivo de los servicios para el control del cáncer que son accesibles al resto de la población. Estos hechos, llevan a la inevitable necesidad de observar estrategias basadas en las características y connotaciones religiosas, culturales, educativas y socio-políticas de las poblaciones indígenas, a fin de comprender e interpretar sus dinámicas y así generar acciones que incrementen el cumplimiento de los objetivos propuestos y un mayor aprovechamiento de los recursos disponibles que en no pocas oportunidades son escasos.

Del mismo modo, la movilización social para el control del cáncer en Colombia, hasta el momento, se ha caracterizado por la poca capacidad de incidir en la toma de decisiones y de sensibilizar a la población respecto a la dimensión del impacto de la enfermedad en el país; se han descrito características propias del contexto socio-político colombiano que están implicadas en estos hechos: a) el interés de la población por movilizarse se centra en necesidades sentidas, lo que dificulta la implementación de acciones alrededor de problemáticas que, como el cáncer, requieren una perspectiva en el largo plazo, b) las movilizaciones asociadas al control del cáncer han sido marginales y se han acompañado por otras demandas que se consideran prioritarias y c) existe una falta de formación en términos de participación (39). 


\section{Sugerencias}

La generación de modelos interculturales respetuosos de las diferencias culturales y con el propósito de desarrollar una política de cooperación entre pueblos indígenas y el Estado (46) permite una mejor gestión en salud con pertinencia cultural, a fin de contribuir al mejoramiento de la calidad de vida de los pueblos indígenas, al fortalecimiento de la medicina indígena y el aumento del acceso y la capacidad resolutiva de una red de servicios interculturales para el país.

En este sentido, para orientar adecuadamente modelos interculturales en los cuales prime el identificar, explicar y comprender la visión del cáncer en los pueblos indígenas se requiere de una estrategia de comunicación educativa y de movilización social que emplee la consulta previa, buscando la participación activa de esta comunidad en la solución de las problemáticas que les afectan en relación con el cáncer.

La condición connatural de una co-creación de carácter intercultural en esta materia, es el desarrollo de metodologías plenamente cualitativas y participativas que resalten el valor de la oralidad y la tradición como elementos centrales de la comunicación de los pueblos indígenas y herramientas de resistencia en la preservación de su cultura y saberes ancestrales.

\section{Referencias}

1.Organización Panamericana de la Salud. La salud de los pueblos indígenas. [Internet] [Consultado el 10 de agosto de 2017]. Disponible en: http://www.paho. org/chi/index.php?option=com_content\&view $=$ article\&id $=83$ : salud-pueblos-indige nas\&Itemid $=213$

2. Organización Panamericana de la Salud. La salud de los pueblos indígenas. [Internet] [Consultado el 10 de agosto de 2017]. Disponible en: http://www.paho. org/chi/index.php?option=com_content\&view=article\&id=83: salud-pueblos-indigenas\&ltemid=213 (la referencia 1 y la 2 son iguales)

3. Naciones Unidas. Pueblos indígenas. [Internet] [Consultado el 11 de agosto de 2017]. Disponible en: http://www.un.org/ es/globalissues/indigenous/
4. Organización Mundial de la Salud. La salud de los pueblos indígenas: Nota descriptiva $\mathrm{N}^{\circ}$ 326. 2007. [Internet] [Consultado el 10 de agosto de 2017]. Disponible en: http://www.who.int/hhr/Fact $\% 20$ Sheet $\% 20$ Indigenous $\% 20 \mathrm{Health} \% 20$ Nov\%202007\%20Final\%20SP.pdf

5. Grupo de Trabajo Internacional sobre Asuntos Indígenas. El Mundo Indígena. 2006. [Consultado el 12 de agosto de 2017]. Disponible en: HYPERLINK "https://www.iwgia.org/images/publications/0533_EL_MUNDO_INDIGENA_2006.pdf"https://www.iwgia.org/ images/publications//0533_EL_MUNDO_ INDIGENA_2006.pdf

6. Gillette H, Patrinos H. Pueblos indígenas, pobreza y desarrollo humano en América Latina: 1994-2004. 2005. [Internet] [Con- 
sultado el 28 de agosto de 2017]. Disponible en: http://documentos.bancomundial. org/curated/es/712501468300536572/ pdf/330200PueblosIndigenasPobreza1resumen1es.pdf

7. Comisión Económica para América Latina y el Caribe. Encuentro latinoamericano y caribeño sobre las personas de edad - Seminario técnico. Sesión III: La situación socioeconómica de la población adulta mayor: un grupo vulnerable. 2000. [Internet] [Consultado el 01 de noviembre de 2017]. Disponible en: https:// www.cepal.org/publicaciones/xml/4/5604/ Icl1399e_s3.pdf

8. Organización Internacional del Trabajo. Convenio Núm. 169 de la OIT sobre Pueblos Indígenas y Tribales. 2014. [Internet] [Consultado el 22 de agosto e 2017]. Disponible en: http://www.ilo.org/wcmsp5/ groups/public/---americas/---ro-lima/documents/publication/wcms_345065.pdf

9. Oficina de la Alta Comisionada de Naciones Unidas para los Derechos Humanos. Guía para la aplicación judicial: Los Derechos de los Pueblos Indígenas en el Convenio 169 de la OIT (Organización Internacional del Trabajo). 2009. [Internet] [Consultado el 17 de agosto de 2017]. Disponible en: http://www.acnur. org/t3/fileadmin/Documentos/Publicaciones/2009/6903.pdf

10. Departamento Administrativo Nacional de Estadística (DANE). Colombia una nación multicultural, su diversidad étnica. 2007. [Internet] [Consultado 22 de agosto de 2017]. Disponible en: http://www.dane. gov.co/files/censo2005/etnia/sys/colombia_nacion.pdf

11. Organización Nacional Indígena de Colombia (ONIC). Campaña Palabra dul$c e$, aire de vida. Forjando caminos para la pervivencia de los pueblos indígenas en riesgo de extinción en Colombia. 2010. [Internet] [Consultado el 26 de agosto de

140 2017]. Disponible en: https://issuu.com/ adminonic/docs/palabra_dulce_aire_de_ vida
12. Organización Nacional Indígena de Colombia. Pueblos Indígenas. 2009. [Internet] [Consultado el 26 de agosto de 2017]. Disponible en: http://www.onic. org.co/noticias/2-sin-categoria/1038-pueblos-indigenas

13. Moore P. S, Forman D, Piñeros M, Fernandez M. S, Oliveira M y Bray F. Cancer in indigenous people in Latin America and the Caribbean: a review. Cancer Medicine. $2014 ; 3(1)$ : 70-80.

14. Organización Panamericana de la Salud (OPS), Ministerio de salud y Protección Social. Perfil epidemiológico de pueblos indígenas en Colombia. 2010. [Internet] [Consultado el 12 de septiembre de 2017]. Disponible en: https://www. minsalud.gov.co/plandecenal/Documents/ Documentos\%20T\%C3\%A9cnicos\%20 Plan\%20Decenal\%20de\%20Salud\%20 P\%C3\%BAblica/Perfil\%20Epidemiol\%C3\%B3gico\%20P.\%20Ind\%C3\%ADgenas $\% 20$ Parte $\% 202$.pdf

15.Woods L, Rachet B, Coleman M. Origins of socio economic inequalities in cancer survival: a review. Annals of Oncology. 2006; 17: 5-19.

16 Suárez, M. Servicios de salud, Pueblos Indígenas y Prácticas Médicas. Simposio: Servicios de Salud, Pueblos Indígenas y Prácticas Médicas. s.f. [Internet] [Consultado el 20 de septiembre de 2017]. Disponible en: http://www.revistas.unal. edu.co/index.php/revsaludpublica/article/ view/18684/19580

17. Corporación Memoria y Saber Popular. Sistema indígena de salud propio intercultural. 2012. [Internet] [Consultado el 20 septiembre de 2017]. Disponible en: http://www.saberpopular.org/index. php?option=com_content\&view=article\&i$d=253$ : sistema-indigena-de-salud-propio-e-intercultural-sispi\&catid $=46 \&$ Itemid $=243$

18. Perafán C, Savedoff W. Los pueblos indígenas y la salud: cuestiones para la 
discusión y el debate. Washington D.C.: Departamento de Desarrollo Sostenible, Bid. 2001.

19. Ley 691 de 2001[Internet] Colombia. Por la cual se reglamenta la participación de los Grupos Étnicos en el Sistema General de Seguridad Social en Colombia. [Consultado el 28 de septiembre de 2017]. Disponible en: http://www.alcaldiabogota.gov.co/sisjur/normas/Norma1. jsp?i=4454

20. Organización Panamericana de la Salud. Interculturalidad en Salud Reproductiva, dónde estamos y cómo estamos. 2010. [Internet] [Consultado el 28 de septiembre de 2017]. Disponible en: http:// bvsper.paho.org/videosdigitales/matedu/20101222_interculturalidad_salud_reproductiva_aguzman.pdf?ua $=1$

21. Rosero J. El derecho a la salud de los Pueblos Indígenas en Colombia, Ecuador y Perú. 2016. [Internet] [Consultado 15 de septiembre de 2017]. Disponible en: http://www.umariana.edu.co/ojs-editorial/ index.php/libroseditorialunimar/article/ view/899/874 http://www.umariana.edu. co/ojs-editorial/index.php/libroseditorialunimar/article/viewFile/899/874

22. Ministerio Federal de Cooperación Económica y Desarrollo de Alemania. El derecho a la consulta previa: Normas jurídicas, prácticas y conflictos en América Latina. sf. [Internet] [Consultado el 30 de octubre de 2017]. Disponible en: https:// www.giga-hamburg.de/sites/default/files/ md_pdf/1303_destradi_konferenzbericht_es.pdf

23. Anaya, J. El deber estatal de consulta a los pueblos indígenas dentro del derecho internacional. Seminario sobre la consulta previa a los pueblos indígenas y el rol de los Ombudsman en América Latina. 2013. [Internet] [Consultado el 20 de octubre de 2017] Disponible en: http://www. politicaspublicas.net/ panel/re/docs/1660-deber-de-consulta-previa.html
24. Departamento Nacional de Planeación. Apuntes sobre la consulta previa con grupos étnicos. 2011. [Internet] [Consultado el 02 de noviembre de 2017]. Disponible en: http://www.mininterior.gov.co/sites/ default/files/noticias/apuntes_sobre_consulta_previa_con_grupos_etnicos.pdf

25. Dinero. 10 pasos para realizar consulta previa en Colombia. 2013. [Internet] [Consultado el 31 de octubre de 2017]. Disponible en: http://www.dinero.com/ actualidad/noticias/articulo/abc-consulta-previa-colombia/189017

26. Corte Constitucional. Sentencia T-080 del 7 febrero de 2017. Derecho Fundamental a la Consulta Previa. [Internet] [Consultado el 01 de noviembre de 2017]. Disponible en: http://www.corteconstitucional.gov.co/relatoria/2017/t-080-17.htm

27 Corte Constitucional. Sentencia C-313 del 29 de mayo 2014. Proyecto de Ley Estatutaria sobre Derecho Fundamental a La Salud. [Internet] [Consultado el 01 de noviembre de 2017]. Disponible en: http:// www.corteconstitucional.gov.co/relatoria/2014/C-313-14.htm

28. Chong A, Roder D. Exploring differences in survival from cancer among Indigenous and non-Indigenous Australians: implications for health service delivery and research. Asian Pac J Cancer. Prev. 2010; 11 (4): 61-953.

29. Centros para el Control y la Prevención de Enfermedades. El cáncer entre los indoamericanos y los nativos de Alaska. 2016. [Internet] [Consultado el 15 de septiembre de 2017]. Disponible en: https://www.cdc.gov/spanish/cancer/healthdisparities/what_cdc_is_doing/aian. $\mathrm{htm}$

30. Ministerio de Salud y Protección Social. Perfil de Salud de la Población Indígena, y medición de desigualdades en salud. Colombia 2016. 2016. [Internet] [Consultado el 15 de septiembre de 2017]. Disponible en: https://www.minsalud.gov. $\mathrm{co} / \mathrm{sites} / \mathrm{rid} /$ Lists/BibliotecaDigital/RIDE/ 
VS/ED/PSP/Perfil-salud-pueblos-indigenas-colombia-2016.pdf

31. Organización Mundial de la Salud. Programas Nacionales de lucha contra el cáncer. Directrices sobre política y gestión. Resumen de orientación. 2002. [Internet] [Consultado 15 de septiembre de 2017]. Disponible en: http://www.who.int/ cancer/media/en/423.pdf

32. Organización Panamericana de la Salud. Una visión de salud intercultural para los pueblos indígenas de las Américas. Washington D.C.: OPS. 2008.

33. Organización Panamericana de la Salud. Iniciativa de Salud de los Pueblos Indígenas. Lineamientos Estratégicos y Plan de Acción 2003 - 2007. [Internet] [Consultado el 02 de octubre de 2017]. Disponible en: http://www1.paho. org/hq/dmdocuments/2009/50\%20EspPlan2003-2007.pdf

34. Constitución Política de Colombia. 1991. Disponible en: http://www.secretariasenado.gov.co/senado/basedoc/constitucion_politica_1991.html (Falta Información)

35. Davinson G, Jélvez I y Yánez S. Investigación del diseño de un programa de salud intercultural para Indígenas. Bases para una propuesta de un Seguro Indígena. 1997. [Internet] [Consultado el 15 de septiembre de 2017]. Disponible en: http://www.estudiosindigenas.cl/images/_ publ/Salud\%20intercultural\%20a\%20indigenas.pdf

36. Vich, V. El Estado está de vuelta: desigualdad, diversidad y democracia. Lima: Instituto de Estudios Peruanos; 2005.

37. Hasen F. Documento Sistematización Reunión Nacional del Consejo Consultivo de Adolescentes y Jóvenes. 2011. [Internet] [Consultado el 11 de septiembre de 2017]. Disponible en: http://www.minsal. gob.cl

38. Aztaiza N, Rodríguez S. Diálogo intercultural en salud: una estrategia para res- catar los saberes y prácticas médicas en torno a la salud maternoinfantil de las comunidades afrocaucanas. Revista Virtual Universidad Católica del Norte. [Internet] 2012;37. Disponible en: http://revistavirtual.ucn.edu.co/

39. Suárez R, Wiesner C, González C, Cortés C, Shinchi A. Antropología del Cáncer e Investigación Aplicada En Salud Pública. Revista de Estudios Sociales. 2004; 17:42-55.

41.Alarcón A, Vidal A, Neira J. Salud intercultural: elementos para la construcción de sus bases conceptuales. Rev. Méd. (Chile) 2003; 131: 1061-1065.

42.Berlin E, Fowkes C. Teaching Framework for Cross-Cultural Health Care. Mayfield Publishing Company. California. 1998.

43. Ministerio de la Protección Social, Instituto Nacional de Cancerología. Guía para la comunicación educativa en el marco del control del cáncer. Bogotá, D.C.: Instituto Nacional de Cancerología. 2008.

44. Hasen F. Interculturalidad en salud: Competencias en prácticas de salud con población indígena. Ciencia y Enfermería. 2012; 18 (3): 17-24.

44. Cunningham, M. Etnia, cultura y salud: La experiencia de la salud intercultural como una herramienta para la equidad en las Regiones Autónomas de Nicaragua. [Internet]. 2002. Disponible en: http://enlaceacademico.ucr.ac.cr/sites/default/files/publicaciones/MCunninghamConf_0.pdf

45. Díaz L, Mueses C. Protesta indígena y movimiento social en Colombia. Apropiación política de la María-Piendamó (Departamento del Cauca, Colombia). C\&P (Colombia) 2011; (2):104-140.

46. Organización Panamericana de la Salud. Salud en las Américas: 2007. Segunda versión. Washington D.C.: OPS. 2007 
47. Organización Panamericana de la Salud. Enfoque intercultural. La prevención de tuberculosis en pueblos indígenas. 2009. [Internet] [Consultado el 02 de noviembre de 2017]. Disponible en: http://www.paho.org/coL/index.php?option=com_docman\&view=download \&category_slug=publicaciones-ops-oms-colombia\&alias=1349-enfoque-intercultural-tb-indigenas\&ltemid $=688$

48. Ministerio de la Protección Social, Agencia canadiense para el Desarrollo Internacional, Organización Panamericana de la salud, Organización Mundial de la
Salud. Experiencia en Colombia de la Estrategia DOTS/TAS para el manejo de la tuberculosis en pueblos indígenas ubicados en los departamentos del Amazonas, Cesar, Nariño, Guainía, Guaviare, la Guajira y Vichada. 2007. [Internet] [Consultado el 12 de octubre de 2017]. Disponible en: http://www.col.ops-oms.org/publicaciones/tuberculosis.pdf

49. Boccara G, Etnogubernamentalidad. La formación del campo de la salud intercultural en Chile. Revista de Antropología Chilena. 2007; 39(2): 185-207. 
